This item was submitted to Loughborough's Institutional Repository (https://dspace.lboro.ac.uk/) by the author and is made available under the following Creative Commons Licence conditions.

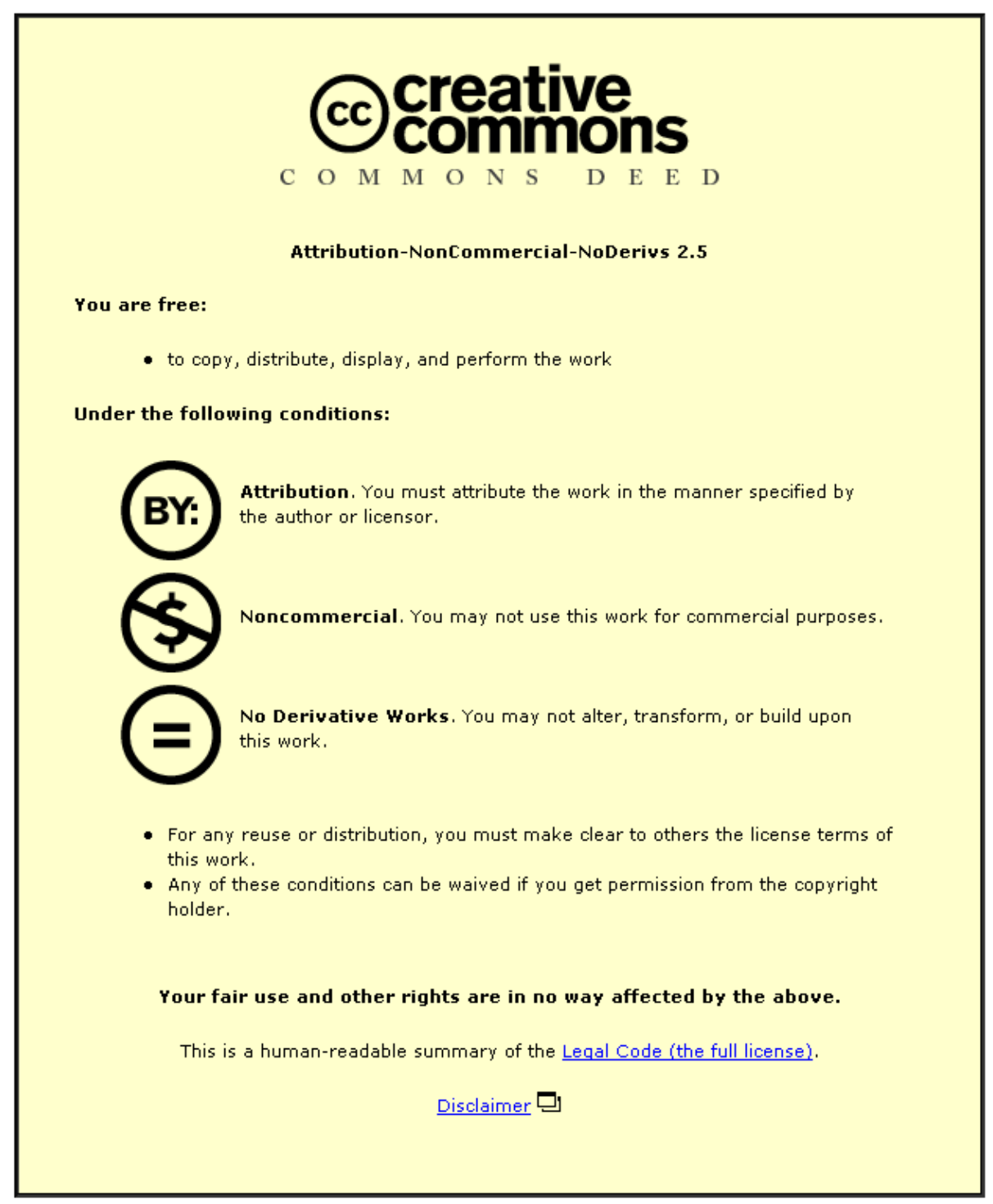

For the full text of this licence, please go to: http://creativecommons.org/licenses/by-nc-nd/2.5/ 


\title{
Modelling the maximum voluntary joint torque / angular velocity relationship in human movement
}

\author{
Maurice R. Yeadon ${ }^{1}$, Mark A. King ${ }^{1}$ and Cassie Wilson ${ }^{2}$ \\ ${ }^{1}$ School of Sport and Exercise Sciences, Loughborough University, LE11 3TU, UK \\ ${ }^{2}$ School of Sport, PE and Recreation, University of Wales Institute Cardiff, CF23 6XD, UK
}

\begin{abstract}
The force exerted by a muscle is a function of the activation level and the maximum (tetanic) muscle force. In "maximum" voluntary knee extensions muscle activation is lower for eccentric muscle velocities than for concentric velocities. The aim of this study was to model this "differential activation" in order to calculate the maximum voluntary knee extensor torque as a function of knee angular velocity. Torque data were collected on two subjects during maximal eccentric-concentric knee extensions using an isovelocity dynamometer with crank angular velocities ranging from $50^{\circ} \mathrm{s}^{-1}$ to $450^{\circ} \mathrm{s}^{-1}$. The theoretical tetanic torque / angular velocity relationship was modelled using a four parameter function comprising two rectangular hyperbolas while the activation / angular velocity relationship was modelled using a three parameter function that rose from submaximal activation for eccentric velocities to full activation for high concentric velocities. The product of these two functions gave a seven parameter function which was fitted to the joint torque / angular velocity data, giving unbiased root mean square differences of $1.9 \%$ and $3.3 \%$ of the maximum torques achieved. Differential activation accounts for the nonhyperbolic behaviour of the torque / angular velocity data for low concentric velocities. The maximum voluntary knee extensor torque that can be exerted may be modelled accurately as the product of functions defining the maximum torque and the maximum voluntary activation level. Failure to include differential activation considerations when modelling maximal movements will lead to errors in the estimation of joint torque in the eccentric phase and low velocity concentric phase.
\end{abstract}

Keywords: muscle, joint velocity, differential activation, maximum velocity knee extension, isovelocity dynamometer

\section{Introduction}

Simulation models of human movement often incorporate representations of muscles that produce torques about the joints and drive the simulations (Alexander, 1990; Hatze, 1981; Pandy and Zajac, 1991). The accuracy of such simulations is dependent upon realistic torques being generated by the muscle representations and this is the focus of the present paper.

The variation in maximum muscle force with the rate of change of muscle length is a fundamental characteristic of skeletal muscle. In the concentric phase tetanic muscle force decreases hyperbolically with increasing speed of shortening to approach zero at maximum shortening velocity (Hill, 1938). In the eccentric phase maximum muscle force increases rapidly to around 1.5 times the isometric value with increasing speed of lengthening and then plateaus for higher velocities (Harry et al., 1990).

In contrast studies of maximum eccentric voluntary contractions of human skeletal muscle show little increase in force above the isometric level (Westing et al., 1988). This suggests that full activation is not achieved in voluntary eccentric contractions. Westing et al. (1990) investigated the effect of supplementing voluntary activation with electrical muscle stimulation during isovelocity knee extension and found that eccentric torque increased by more than $20 \%$ while concentric torque appeared to be unchanged. Subsequently Westing et al. (1991) measured mean full-wave rectified EMG on three knee extensors during maximum 
voluntary isovelocity eccentric and concentric knee extensions. They found that EMG activity was lower under eccentric loading than concentric loading and did not appear to change across eccentric velocities, while EMG increased with increasing concentric velocities. It was speculated that this differential innervation with joint (and muscle) velocity was an injury protection mechanism that resulted in depressed activation and submaximal forces under eccentric conditions.

It may be expected, therefore, that the maximum voluntary torque is a function of the theoretical tetanic torque at a given joint angular velocity and a differential activation function that increases from a depressed level for high eccentric velocities to full activation for high concentric velocities. The aim of this study is to present a method for modelling this differential activation profile in order to calculate the maximum voluntary joint torque over the full range of joint angular velocities for use in simulation models of human movement.

\section{Method}

Isovelocity torque data were collected and used to calculate torque parameters for the knee by fitting a seven parameter function to the data in order to express joint torque as a function of joint angular velocity.

\section{Data Collection}

An active isovelocity dynamometer (Cybex NORM) was used to collect knee extension data (crank angle, crank angular velocity and crank torque) on two subjects while joint angle data were collected via a goniometer. Subject 1 was an international male high jumper of height $1.89 \mathrm{~m}$ and mass $82 \mathrm{~kg}$ while Subject 2 was an elite martial artist of height $1.78 \mathrm{~m}$ and mass $90 \mathrm{~kg}$.

The exercise protocol for each knee extension trial comprised two repetitions of a concentric-eccentric exercise over a $90^{\circ}$ range of crank motion at a preset crank angular velocity. This protocol was chosen with the intention to only use the torque data produced during the central eccentric-concentric phase of each trial. Performing contractions before and after this central eccentric-concentric phase of interest ensured that the subject was performing maximally during this central phase. The sequence of crank angular velocities of the concentric-eccentric trials was $50^{\circ} \mathrm{s}^{-1}, 100^{\circ} \mathrm{s}^{-1}, 150^{\circ} \mathrm{s}^{-1}, 200^{\circ} \mathrm{s}^{-1}, 250^{\circ} \mathrm{s}^{-1}, 300^{\circ} \mathrm{s}^{-1}$, $350^{\circ} \mathrm{s}^{-1}, 400^{\circ} \mathrm{s}^{-1}$ and $450^{\circ} \mathrm{s}^{-1}$. This sequence of increasing crank angular velocities was chosen in preference to a randomised set since adoption of the latter procedure could have resulted in less than maximal performance at high angular velocities. It should be noted that maximal efforts at low concentric velocities were physically demanding as the time period was long and that maximum torques were difficult to achieve at high eccentric velocities. The athletes gave informed consent for the procedures, which were carried out in accordance with the protocol approved by Loughborough University Ethical Advisory Committee.

Synchronous crank torque and joint angle data for each trial were recorded at $1000 \mathrm{~Hz}$ to computer file via an analog-digital converter and were converted into the appropriate units using calibration data. The isovelocity portions of the central eccentric-concentric phase of each trial were identified by manually inspecting the joint angle time history and subsequently the data files of the time histories of the crank torque and the joint angle data were edited to leave just these portions.

For all the trials joint angle was linearly regressed against time for the isovelocity phases and joint angular velocity was determined. The crank torque data recorded by the dynamometer were then corrected for both foot and shank weight as well as for crank arm weight. Joint torque was calculated from corrected crank torque in a similar way to King and Yeadon (2002) using: 


$$
\mathrm{T}_{\mathrm{j}}=\mathrm{T}_{\mathrm{c}} \cdot \frac{\mathrm{d}_{\mathrm{j}}}{\mathrm{d}_{\mathrm{c}}}
$$

where: $\mathrm{T}_{\mathrm{c}}=$ crank torque, $\mathrm{T}_{\mathrm{j}}=$ joint torque, $\mathrm{d}_{\mathrm{c}}=$ crank moment arm, $\mathrm{d}_{\mathrm{j}}=$ joint moment arm. This correction assumes that the reaction forces at the cuff interface between shank and crank arm are approximately perpendicular to both shank and crank. Under high loading the thigh moves relative to the cushioned seat support and the shank moves relative to the crank arm causing a difference in the moment arms of the reaction forces about the crank and knee centres. $d_{j}$ and $d_{c}$ were calculated from digitised video of the movement.

The maximum and average joint torque at each angular velocity were found. The maximum torque values were regressed against the average torque values and were replaced by the regression values to give a 'maximal' data set. This resulted in a set of 18 "maximal" joint torques and joint angular velocities that were less noisy than the original maximum torque values and were independent of joint angle.

\section{Torque Model}

A torque model was developed using a four parameter function to express maximum joint torque at full activation as a function of joint angular velocity. Two hyperbolic functions, one representing the concentric phase and one representing the eccentric phase were used to express the maximum torque at full activation as a function of angular velocity. The hyperbola representing the concentric phase was a rotational equivalent of the classic hyperbola of Hill (1938) and an inverted rectangular hyperbola was used to represent the eccentric phase. The function was defined by four parameters: the maximum torque $T_{\max }$ in the eccentric phase, the isometric torque $T_{0}$, the angular velocity $\omega_{\max }$ at which the curve reaches zero torque, and $\omega_{\mathrm{c}}$ defined by the vertical asymptote $\omega=-\omega_{\mathrm{c}}$ of the Hill hyperbola (Figure 1).



Figure 1. The four parameter maximum torque function comprising branches of two rectangular hyperbolas with asymptotes $\mathrm{T}=-\mathrm{T}_{\mathrm{c}}$ and $\omega=-\omega_{\mathrm{c}}$, and $\mathrm{T}=\mathrm{T}_{\max }$ and $\omega=\omega_{\mathrm{e}}$.

In the concentric phase the relationship between $\mathrm{T}$ and $\omega$ was given by the classic Hill hyperbola:

$$
\mathrm{T}=\frac{\mathrm{C}}{\left(\omega_{\mathrm{c}}+\omega\right)}-\mathrm{T}_{\mathrm{c}} \quad(\text { if } \omega \geq 0)
$$

where: $\mathrm{T}_{\mathrm{c}}=\mathrm{T}_{0} \omega_{\mathrm{c}} / \omega_{\max }, \mathrm{C}=\mathrm{T}_{\mathrm{c}}\left(\omega_{\max }+\omega_{\mathrm{c}}\right)$. 
In the eccentric phase the relationship between $\mathrm{T}$ and $\omega$ was given by the rectangular hyperbola:

$$
\mathrm{T}=\frac{\mathrm{E}}{\left(\omega_{\mathrm{e}}-\omega\right)}+\mathrm{T}_{\max } \quad(\text { if } \omega \leq 0)
$$

where: $\omega_{\mathrm{e}}=\frac{\left(\mathrm{T}_{\max }-\mathrm{T}_{0}\right)}{\mathrm{kT}_{0}} \cdot \frac{\omega_{\max } \omega_{\mathrm{c}}}{\left(\omega_{\max }+\omega_{\mathrm{c}}\right)}, \mathrm{E}=-\left(\mathrm{T}_{\max }-\mathrm{T}_{0}\right) \omega_{\mathrm{e}}$ and $\mathrm{k}=$ ratio of the slopes of the eccentric and concentric functions at $\omega=0$. The value of $\mathrm{k}$ was set at 4.3 , the theoretical value which Huxley (1957) predicted with his original model.

\section{Differential Activation}

An additional three parameter function was used to define the differential activation. The three parameters were: the lowest level of activation $a_{\min }$ in the eccentric phase, the angular velocity $\omega_{1}$ at the point of inflection of the function (Figure 2), and a parameter $m$ that governed the rate at which the activation increases with angular velocity $(1 / \mathrm{m}$ was proportional to the slope at the point of inflection). The maximum activation level $\mathrm{a}_{\max }$ was assumed to be equal to 1.0. The differential activation was defined by equation (4) which can be used to express a as a function of $\omega$ using the quadratic formula.

$$
\omega-\omega_{1}=\frac{+m\left(a-0.5\left(a_{\min }+a_{\max }\right)\right)}{\left(a_{\max }-a\right)\left(a-a_{\min }\right)}
$$

where: $\mathrm{a}=$ activation level and $\omega=$ angular velocity.

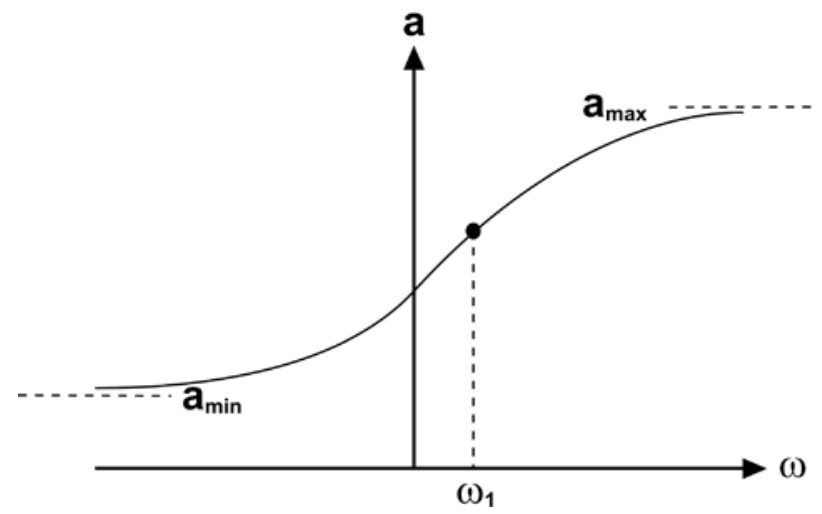

Figure 2. The three parameter differential activation function in which the activation a rises from $a_{\min }$ to $a_{\max }$ with a point of inflection at $\omega=\omega_{1}$.

\section{Seven Parameter Function}

The four parameter function defining maximum torque was then multiplied by the three parameter function defining differential activation to give a seven parameter function representing the maximum voluntary torque. The seven parameters defining the two functions were calculated using the Simulated Annealing optimisation algorithm (Corana et al., 1987) to minimise the unbiased root mean square (RMS) difference between the function values and the known maximal torques. Simulated Annealing was used with various initial conditions, parameter order and random number seeds to ensure that the closest fit to the data was obtained. Each of the parameters was allowed to vary between upper and lower limits. The lower limit of $\mathrm{T}_{0}$ was set to the average of the joint torque values corresponding to crank angular velocities of $50^{\circ} \mathrm{s}^{-1}$ and $-50^{\circ} \mathrm{s}^{-1}$ and the upper limit was set $20 \%$ larger than this. The lower limit of $\omega_{\max }$ for each subject was set at the highest knee extension velocity in a recorded running jump for height by Subject $1\left(770^{\circ} \mathrm{s}^{-1}\right.$ or $\left.13.4 \mathrm{rad}_{\mathrm{s}} \mathrm{s}^{-1}\right)$ and the upper limit was set at twice this value. $\omega_{\mathrm{c}}$ was allowed to vary between 1 and $100 \mathrm{rad} . \mathrm{s}^{-1}, \mathrm{~m}$ was required to 
be positive, $a_{\min }$ was allowed to vary between 0 and 1 , and finally $\omega_{1}$ was allowed to vary between $-350^{\circ} \mathrm{s}^{-1}$ and $350^{\circ} \mathrm{s}^{-1}\left(-6\right.$ to +6 rad. $\left.\mathrm{s}^{-1}\right)$. The value of $\mathrm{T}_{\max }$ was set at 1.5 times the value of $\mathrm{T}_{0}$ and was therefore not considered to be a free parameter.

In order to estimate the likely error in each parameter the torque deviations from the seven parameter fit were calculated by subtracting the fitted torque value from the maximal torque at each angular velocity. This resulted in nine torque deviations for the eccentric velocities and nine torque deviations for the concentric velocities. The eccentric and concentric deviation sets were each permuted cyclically to give eight new sets of deviations. These deviations were then added to the fitted torque values at the corresponding angular velocities to generate eight new maximal torque / angular velocity data sets which were used to produce eight new seven parameter fits. The standard deviation from the nine estimates of a parameter was used as an error estimate of that parameter.

In addition to the determination of the seven parameter function, a four parameter function (no differential activation) was also fitted to the torque data. The parameters were determined using Simulated Annealing to minimise the unbiased RMS difference between the function values and the known maximum torques. There were only two free parameters as $\mathrm{T}_{0}$ was set equal to the average of the torques corresponding to crank velocities of $\pm 50^{\circ} \mathrm{s}^{-1}$ and again $\mathrm{T}_{\max }$ was fixed at 1.5 times the value of $\mathrm{T}_{0}$.

\section{Results}

The seven parameter fit defining the torque / angular velocity relationship for Subject 1 and Subject 2 (Table 1) resulted in unbiased RMS fit errors of $1.9 \%$ and $3.3 \%$ of maximum torque respectively (Figures 3a, 4a). The estimated error in each parameter is shown in Table1.

Table 1. Torque parameters and their error estimates for the seven parameter function

\begin{tabular}{|c|c|c|}
\hline parameter & Subject 1 & Subject 2 \\
\hline $\mathrm{T}_{\max }$ & $437 \pm 13 \mathrm{Nm}$ & $455 \pm 10 \mathrm{Nm}$ \\
\hline $\mathrm{T}_{0}$ & $291 \pm 9 \mathrm{Nm}$ & $303 \pm 7 \mathrm{Nm}$ \\
\hline$\omega_{\max }$ & $13.4+0.1 \mathrm{rad} . \mathrm{s}^{-1}$ & $26.8-5.1 \mathrm{rad} . \mathrm{s}^{-1}$ \\
\hline$\omega_{\mathrm{c}}$ & $51.4 \pm 19.6 \mathrm{rad} . \mathrm{s}^{-1}$ & $8.7 \pm 5.6 \mathrm{rad} . \mathrm{s}^{-1}$ \\
\hline $\mathrm{a}_{\min }$ & $0.66 \pm 0.03$ & $0.72 \pm 0.02$ \\
\hline $\mathrm{m}$ & $0.35 \pm 0.13$ & $0.21 \pm 0.12$ \\
\hline$\omega_{1}$ & $0.64 \pm 0.30 \mathrm{rad} . \mathrm{s}^{-1}$ & $-0.5 \pm 0.1 \mathrm{rad} . \mathrm{s}^{-1}$ \\
\hline
\end{tabular}





Figure 3. (a) Seven parameter maximum voluntary torque function for Subject 1 with a root mean square difference of $1.9 \%$, (b) four parameter maximum torque function for Subject 1, (c) three parameter differential activation function for Subject 1. 



Figure 4. (a) Seven parameter maximum voluntary torque function for Subject 2 with a root mean square difference of 3.3\%, (b) four parameter maximum torque function for Subject 2, (c) three parameter differential activation function for Subject 2.

The four parameter fit determined using the same raw data (Table 2) resulted in much higher unbiased RMS percentage differences of $24.5 \%$ (Figure 5) and $21.2 \%$ for the two subjects.

Table 2. Torque parameters for the four parameter function

\begin{tabular}{|c|c|c|}
\hline & Subject 1 & Subject 2 \\
\hline $\mathrm{T}_{\max }$ & $366 \mathrm{Nm}$ & $451 \mathrm{Nm}$ \\
\hline $\mathrm{T}_{0}$ & $244 \mathrm{Nm}$ & $301 \mathrm{Nm}$ \\
\hline$\omega_{\max }$ & $16.1 \mathrm{rad} . \mathrm{s}^{-1}$ & $13.4 \mathrm{rad} . \mathrm{s}^{-1}$ \\
\hline$\omega_{\mathrm{c}}$ & $5.4 \mathrm{rad} . \mathrm{s}^{-1}$ & $54.0 \mathrm{rad} . \mathrm{s}^{-1}$ \\
\hline
\end{tabular}




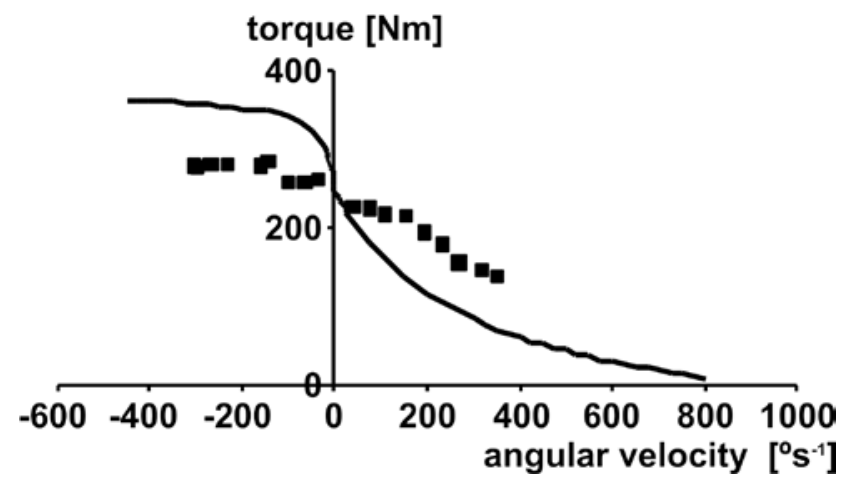

Figure 5. Four parameter function fit for Subject 1 passing through $\mathrm{T}_{0}$ with $\mathrm{T}_{\max }=1.5 \mathrm{~T}_{0}$. The fitted function is too large for eccentric velocities and too small for concentric velocities.

\section{Discussion}

The seven parameter function produced torque / angular velocity profiles that closely matched the isovelocity data for the two subjects (Figures 3a, 4a). The $a_{\min }$ values for the activation level at high eccentric velocities were similar (66\% and $72 \%)$ and the inflection points of the differential activation profile both occurred near an angular velocity of zero (Table 1, Figures 3c, 4c). These values for $\mathrm{a}_{\min }$ are consistent with the results of Westing et al. (1991) who observed reduced EMG activity values in the range $69 \%$ - 85\% for eccentric velocities of $360^{\circ} \mathrm{s}^{-1}$ compared to $360^{\circ} \mathrm{s}^{-1}$ concentric velocities. They are also consistent with the results of Westing et al. (1990) who found that maximum voluntary eccentric torque was $81 \%-83 \%$ of that obtained when tolerable electrical stimulation was superimposed. Since the level of electrical stimulation was limited for the comfort of the participants, these values may overestimate the level of maximum voluntary eccentric torque relative to tetanic eccentric torque.

In order to implement the torque / angular velocity function in a simulation model, an activation time history profile can be multiplied by the four parameter tetanic function to give a torque time history. Alternatively and equivalently the activation time history may be divided by the corresponding differential activation level to give a relative activation profile which is then multiplied by the seven parameter function. This relative activation profile should lie between 0 and 1 . In cases of maximal effort the relative activation profile will ramp up from some initial level to 1.0. In cases of submaximal effort the relative activation profile will remain less than 1.0 throughout. Thus the seven parameter function may be used for both maximal and submaximal activities so long as there is a basis for generating an appropriate relative activation profile.

Depressed activation in the eccentric phase is likely to be based on feed-forward expectations of how the joint angular velocity is likely to change. For example when landing from a jump it is expected that the knee extensors will be eccentrically loaded initially and so the stimulus to the muscles will be submaximal. If the unexpected occurs then the seven parameter formulation may not be appropriate since depressed activation may not occur. In the study of Grabiner and Owings (2003) participants expecting to perform a maximal concentric contraction had an eccentric contraction imposed upon them. It was found that the initial EMG measured during the isometric phase preceding the onset of dynamometer motion resembled that of a concentric contraction, being some $26 \%$ larger than the initial level in an expected eccentric contraction. In such a case a different kind of activation profile should be used in conjunction with the four parameter tetanic function. 
The value of 1.5 for the ratio of maximum eccentric torque to isometric torque in the four parameter tetanic function lies in the middle of the range $1.2-1.9$ obtained for in vitro preparations (Webber and Kriellaars, 1997). It could be argued that the value of 1.4 indicated by the data of Dudley et al. (1990) would be more appropriate since it based on in vivo knee extensor torques of human subjects. The particular value used for this constant will not affect the overall seven parameter function greatly although it will affect the level of $\mathrm{a}_{\min }$.

The four parameters describing the underlying tetanic torque / angular velocity function had similar strength constants $\left(\mathrm{T}_{\max }\right.$ and $\left.\mathrm{T}_{0}\right)$ but quite different velocity constants $\left(\omega_{\max }\right.$ and $\omega_{c}$ ) as shown in Table 1. For Subject $1 \omega_{\max }$ was equal to the lower bound of 13.4 rad.s ${ }^{-1}$ set in the optimization procedure while for Subject $2 \omega_{\max }$ was equal to the upper bound of 26.8 rad. $s^{-1}$. This difference is likely to be a consequence of having no torque data at joint angular velocities above 6 rad.s $\mathrm{s}^{-1}$. The three data points corresponding to high concentric velocities in Figure 3a suggest that a higher value for $\omega_{\max }$ might be more appropriate and that setting a higher lower bound in the optimisation procedure might reduce this problem. While the higher $\omega_{\max }$ for Subject 2 gives a higher torque value at high concentric angular velocities, the lower $\omega_{\mathrm{c}}$ value (8.7 rad.s ${ }^{-1}$ compared to $51.4 \mathrm{rad}_{\mathrm{s}}{ }^{-1}$ ) results in greater curvature of the concentric hyperbola. This gives a lower torque at low concentric angular velocities, offsetting the effect of the high $\omega_{\max }$ value to some extent (Figures $3 \mathrm{~b}, 4 \mathrm{~b}$ ).

The error level of $2 \%-3 \%$ in the torque data produced error estimates of similar magnitude in $\mathrm{a}_{\min }, \mathrm{T}_{\max }$ and $\mathrm{T}_{0}$ (Table 1). $\omega_{\max }$ remained near the lower bound of $13.4 \mathrm{rad} . \mathrm{s}^{-1}$ for Subject 1 while it showed high variability for Subject 2 as a consequence of the limited torque data at high angular velocities. $\omega_{\mathrm{c}}$ exhibited high variability although this would be smaller for Subject 1 if a higher lower bound were imposed on $\omega_{\max }$. The inflection velocity $\omega_{1}$ of the three parameter differential activation function took values close to zero for both subjects with fairly small absolute error estimates while the parameter $m$ that governs the slope of the transition had reasonably large error estimates. Despite the variation in parameter values the graphs of both the four parameter tetanic function and the three parameter differential activation function changed very little for the different parameter sets. Establishing appropriate lower and upper bounds for $\omega_{\max }$ would offset the lack of high velocity concentric torque data to some extent.

When a four parameter function was fitted to the torque / angular velocity data with $\mathrm{T}_{\max }$ set at 1.5 times $\mathrm{T}_{0}$ the fits were unrealistic with RMS errors of more than $20 \%$ (Figure 5). The $T_{0}$ value for Subject 1 was $244 \mathrm{Nm}$ compared to $291 \mathrm{Nm}$ for the $\mathrm{T}_{0}$ value in the seven parameter fit. On the other hand $\mathrm{T}_{0}$ for Subject 2 was $301 \mathrm{Nm}$, only slightly smaller than the $303 \mathrm{Nm}$ from the seven parameter fit. This difference may be understood by comparing the differential activation parameters of the two subjects. Subject 2 started at a higher activation level in the eccentric phase $\left(\mathrm{a}_{\min }=0.72\right.$ compared to 0.66$)$ and this started to rise earlier $\left(\omega_{1}=\right.$ -0.5 rad. $\mathrm{s}^{-1}$ compared to 0.6 rad. $\mathrm{s}^{-1}$ ) and faster $(\mathrm{m}=0.21$ compared to 0.35 ). Consequently at a joint angular velocity of zero the activation level of Subject 2 was 0.90 compared to a level of 0.78 for Subject 1 . The activation level for Subject 2 had effectively reached maximum at a lower concentric velocity than for Subject 1 (Figures 3c, 4c). As a consequence the seven parameter function of Subject 1 shows a pronounced plateau for low concentric velocities whereas that of Subject 2 is closer to a hyperbola with concave curvature for all concentric velocities (Figures 3a, 4a). This plateau in the torque data of Subject 1 at low concentric velocities is evident in previous studies (Dudley et al., 1990; James et al., 1994; King and Yeadon, 2002) and a seven parameter function is required in order to fit such data reasonably. On the other hand the data of Subject 2 shows no such plateau and in such a case a reasonable fit should be possible using a five parameter function in which the maximum eccentric to isometric torque ratio is a free parameter. 
Simulation models that use representations of force-velocity relationships, which neglect differential activation effects, may give unrealistic results for maximal movements where both eccentric and concentric muscle action is involved. For simulations of walking (Pandy and Anderson, 2000) such errors are unlikely to cause problems since activations are submaximal. For movements which require primarily concentric muscle action such as squat jumps (van Soest et al., 1993) there will be maximal activation only for higher concentric velocities and the Hill relationship may be adequate providing appropriate muscle parameters are used. In the case of countermovement jumps (Nagano et al., 2003) in which eccentric muscle action is followed by concentric action there is likely to be more of a problem since maximum voluntary activation may be reached during the eccentric phase or in the low velocity concentric phase. For dynamic movements such as high jumping (Alexander, 1990), long jumping (Hatze, 1981), and perhaps running (Gerritsen et al., 1995), in which there is an impact phase with high eccentric requirements, it will be essential to include an appropriate torque / angular velocity relationship within a simulation model in order to obtain realistic results. To achieve this it will be necessary to incorporate a differential activation profile within the force or torque generation capabilities of the model either explicitly or implicitly, as in the case of Yeadon and King (2002) who used a six parameter exponential function to describe the torque / angular velocity relationship at each joint.

\section{References}

Alexander, R.M., 1990. Optimum take-off techniques for high and long jumps. Philosophical Transactions of The Royal Society B329, 3-10.

Corana, A., Marchesi, M., Martini, C., Ridella, S., 1987. Minimising multimodal functions of continuous variables with the "simulated annealing" algorithm. ACM Transactions on Mathematical Software 13, 262-280.

Dudley, G.A., Harris, R.T., Duvoisin, M.R., Hather, B.M., Buchanan, P., 1990. Effect of voluntary vs. artificial activation on the relationship of muscle torque to speed. Journal of Applied Physiology 69, 2215-2221.

Gerritsen, K.G.M., van den Bogert, A.J., Nigg, B.M., 1995. Direct dynamics simulation of the impact phase in heel-toe running. Journal of Biomechanics 28, 661-668.

Grabiner, M.D., Owings, T.M., 2003. Intent-related differences in surface EMG of maximum eccentric and concentric contractions. Journal of Applied Biomechanics 19, 99-105.

Harry, J.D., Ward, A.W., Heglund, N.C., Morgan, D.L., McMahon, T.A., 1990. Crossbridge cycling theories cannot explain high-speed lengthening behaviour in frog muscle. Biophysical Journal 57, 201-208.

Hatze, H., 1981. A comprehensive model for human motion simulation and its application to the takeoff phase of the long jump. Journal of Biomechanics 14, 135-142.

Hill, A.V., 1938. The heat of shortening and the dynamic constants of muscle. Proceedings of the Royal Society Series B 126, 136-195.

Huxley, A.F., 1957. Muscle structure and theories of contraction. Progress in Biophysics and Biophysical Chemistry 7, 225-318.

James, C., Sacco, P., Hurley, M.V., Jones, D.A., 1994. An evaluation of different protocols for measuring the force-velocity relationship of the human quadriceps muscles. European Journal of Applied Physiology 68, 41-47.

King, M.A., Yeadon, M.R., 2002. Determining subject-specific torque parameters for use in a torque-driven simulation model of dynamic jumping. Journal of Applied Biomechanics 18, 207-217. 
Nagano, A., Komura, T., Himeno, R., Fukashiro, S. 2003. Optimal digital filter cutoff frequency of jumping kinematics evaluated through computer simulation. International Journal of Sport and Health Science 1, 196-201.

Pandy, M.G., Anderson, F.C., 2000. Dynamic simulation of human movement using largescale models of the body. Phonetica 57, 219-228.

Pandy, M.G., Zajac, F.E., 1991. Optimal muscular coordination strategies for jumping. Journal of Biomechanics 24, 1-10.

van Soest, A.J., Schwab, A.L., Bobbert, M.F., van Ingen Schenau, G.J., 1993. The influence of the biarticularity of the gastrocnemius muscle on vertical jumping achievement. Journal of Biomechanics 26, 1-8.

Webber, S., Kriellaars, D., 1997. Neuromuscular factors contributing to in vivo eccentric moment generation. Journal of Applied Physiology 83, 40-45.

Westing, S.H., Cresswell, A.G., Thorstensson, A., 1991. Muscle activation during maximal voluntary eccentric and concentric knee extension. European Journal of Applied Physiology 62, 104-108.

Westing, S.H., Seger, J.Y., Karlson, E., Ekblom, B., 1988. Eccentric and concentric torquevelocity characteristics of the quadriceps femoris in man. European Journal of Applied Physiology 58, 100-104.

Westing, S.H., Seger, J.Y. and Thorstensson, A., 1990. Effects of electrical stimulation on eccentric and concentric torque-velocity relationships during knee extension in man. Acta Physiologica Scandinavica 140, 17-22.

Yeadon, M.R. and King, M.A., 2002. Evaluation of a torque driven simulation model of tumbling. Journal of Applied Biomechanics 18, 195-206. 